\title{
Evaluation of Kolmogorov - Smirnov Test and Energy Detector Techniques for Cooperative Spectrum Sensing in Real Channel Conditions
}

\author{
Demian Lekomtcev, Student Member IEEE, and Roman Marsalek, Member IEEE
}

\begin{abstract}
The cognitive radio technology allows solving one of the main issues of current wireless communication technologies, namely a deficit of vacant spectrum. A dynamic spectrum access used in the cognitive radio networks (CRN) gives an ability to access an unused spectrum in real time. Cooperative spectrum sensing is the most effective method for spectrum holes detecting. It combines sensing information of multiple cognitive radio users. In this paper, an experimental evaluation of spectrum sensing methods based on the Kolmogorov - Smirnov statistical test and Energy Detector using the Universal Software Radio Peripheral (USRP) devices synchronized through a MIMO cable and with further processing in the GNU Radio and Matlab software are presented. Three hard decision fusion schemes are analyzed. Simulation comparison between these rules is presented via Receiver Operating Characteristic (ROC) curves. The influence of real channel with interferences is compared in contrast to commonly assumed AWGN channel model of vacant channel noise.
\end{abstract}

Keywords - Spectrum sensing, K-S test, Energy Detector, Fusion Rules, Receiver Operating Characteristic (ROC), GNU Radio, USRP, MIMO.

\section{INTRODUCTION}

$\mathrm{E}$ ARLIER this year, the IEEE Standards Association announced the creation of "IEEE forms study group to explore standardization for spectrum occupancy sensing technology" [1]. The main objective for this working group is optimizing usage of radio spectrum for wireless broadband services for the IEEE 802.22 standard. That is why, despite the fact that there are many investigations aimed at studying the spectrum sensing, this question is

Paper received April 2, 2015; revised June 15, 2015; accepted June 22 , 2015. Date of publication July 15, 2015. The associate editor coordinating the review of this manuscript and approving it for publication was Prof. Branimir Reljin.

This paper is a revised and expanded version of the paper presented at the 21th Telecommunications Forum TELFOR 2014.

This research has been supported by the MEYS of the Czech Republic project LD12006, with the corresponding COST action IC1004. Thanks also for partial support of the BUT Internal project FEKT-S-14-2177 (PEKOS). The measurements were performed in the laboratories of the SIX center, registration number CZ.1.05/2.1.00/03.0072, the operational program Research and Development for Innovation built with the help of a project CZ.1.07/2.3.00/20.0007 WICOMT.

D. Lekomtcev is with the Department of Radio Electronics, Brno University of Technology, Technicka 12, 61600 Brno, Czech Republic (phone: 420-541146541; e-mail: xlekom00@stud.feec.vutbr.cz).

R. Marsalek is now with the Department of Radio Electronics, Brno University of Technology, Technicka 12, 61600 Brno, Czech Republic (phone: 420-541146582; e-mail: marsaler@feec.vutbr.cz). still open.

The simplest and the most common method of spectrum sensing is the Energy Detector (ED). Moreover the sensing devices do not need any knowledge about the Primary User (PU) signals. The Kolmogorov-Smirnov (K-S) test [2] used in statistics to compare two random distributions is one of the promising approaches to spectrum sensing. Its application to this domain is based on the idea of testing the measured signal distribution over the noise distribution (usually AWGN). In this paper we want to perform an extended analysis of K-S test and ED in more realistic conditions - e.g. to test what is the influence of real vacant channel noise with interferences and to evaluate the influence of the precision of cumulative distribution function estimation. Moreover we are also focusing on few simple hard decision methods for cooperative sensing because these technologies are effective, simple and lowcost to be implemented. The evaluation is based on the experimental data from the Universal Software Radio peripheral (USRP) devices controlled by GNU Radio software with subsequent analysis in Matlab.

It is also important to note that most of the research efforts on different fusion rules for cooperative sensing are analytical and/or Matlab simulations based [3]-[5]. Recently a few measurement-based studies on cooperative sensing can be found in [6]-[8]. In [2], [9] and [10] the authors investigate an individual sensing based on K-S test. These works provide the basis for our paper.

The rest of this paper is organized as follows. In Sections II and III, we introduce K-S test-based and ED based spectrum sensing algorithms, respectively. Section IV briefly presents three most common hard fusion rules for cooperative sensing. In Section $\mathrm{V}$, we provide a description of the experimental setup. Section VI presents simulation results, while Section VII concludes the paper.

\section{SPectrum Sensing BASEd On KOLMOGOROV- SMIRNOV TEST}

The detection of a signal within a noisy measure over a specific frequency band is the key problem associated with spectrum sensing. The essence of this problem is to distinguish between the following two hypotheses:

$$
r(t)=\left\{\begin{array}{lc}
n(t), & H_{0} \\
s(t)+n(t), & H_{1}
\end{array}\right.
$$


where $r(t)$ is the received signal at Secondary User (SU) location, $s(t)$ represents the transmitted signal of the PU observed at SU, and $n(t)$ is a noise (in the simplest case the AWGN, or other noise model).

In the case of K-S test, a decision between one or the other hypothesis is performed as described below. This test checks the accordance between the empirical (measured) and the theoretical distribution functions, whereas the distribution parameters of the theoretical distribution are known in advance. Fig. 1 illustrates the principle of this method.

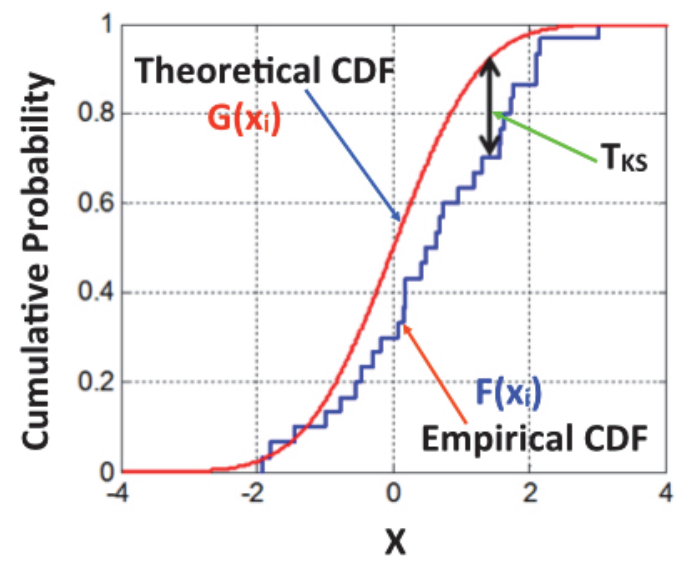

Fig. 1. Visual description of K-S test.

The null hypothesis $H_{0}$ is assumed to be valid when the empirical and theoretical probability distributions are not statistically significantly different and the alternative hypothesis $H_{1}$ is assumed when these distribution are significantly different. First, the empirical Cumulative Distribution Function (CDF), i.e., CDF of the received signal is estimated.

This function is defined by:

$$
F(x)=\frac{1}{N} \sum_{\mathrm{n}=1}^{\mathrm{N}} J(x(n) \leq x)
$$

where $J$ denotes the indicator function, which takes on a value " 1 " if the input is true, and a value " 0 " otherwise, $N$ denotes the number of data samples from each given signal segment. The largest absolute difference between the empirical and the theoretical CDFs is used as the goodness-of-fit statistic, given by [11]:

$$
T_{K S}=\sup _{x \in R}\{F(x)-G(x) \mid\}
$$

where $G(x)$ is the known (expected) theoretical CDF. In accordance with [11] this equation can be expressed as:

$$
T_{K S}=\max _{i}\left\{\left|F\left(x_{i}\right)-G\left(x_{i}\right)\right|\right\}
$$

where $F\left(x_{i}\right)$ denotes the value of CDF evaluated at the point $x_{i}$.

To calculate the theoretical CDF for the absolute value of complex signal and additive white noise with real and imaginary parts having normal distribution, the chi-square distribution with two degrees of freedom is used [12]. In Matlab this can be implemented by calling the function:

$$
G\left(x_{i}\right)=\operatorname{chi} 2 \operatorname{cdf}\left(x_{i}, v\right),
$$

that computes the chi-square $\mathrm{CDF}$ at each of the values in $x_{i}$ using the corresponding degrees of freedom $v$ (in our case $v=2$ ).

The hypothesis $H_{1}$ is accepted at the significance level $\alpha$ (the false alarm probability) when the value of the test statistic $T_{K S}$ is lower than a critical value $k(\alpha, N)$. There are tables for the values of $k(\alpha, N)$ for $5 \leq N \leq 50$ in the literature. As in our investigation $N \geq 50$ the critical values can be approximated as [2]:

$$
\mathrm{k}(\alpha, N)=\sqrt{\frac{1}{2 \cdot N} \ln \left(\frac{2}{\alpha}\right)} .
$$

The motivation for this paper was to investigate what is the influence of noise having the distribution different from the exact Gaussian (i.e. real measured noise), to explore the influence of CDF approximation by finite (and small) numbers of points (important for the efficient implementation) and to check the potential of cooperation between several (at least two) sensing nodes.

\section{Spectrum Sensing Based On EnERgy Detector}

In the case of ED technique, spectrum sensing is performed as follows. First $N_{\text {samples }}$ of the received signal from the investigated channel are received by SU. Then the signal is divided into $N_{\text {segments. }}$ Then the signal energy for each segment is estimated e.g. as:

$$
Y=\frac{1}{N} \sum_{n=1}^{N}(r(t))^{2}
$$

where $Y$ represents the output of the energy detector which serves as the test statistic. To make a decision about the presence or absence of a signal, we introduce $\gamma$ as the threshold that varies depending on the noise variance $\sigma_{n}^{2}$. The probability of false alarm $\left(P_{f a}\right)$ and detection probability $\left(P_{d}\right)$ for ED is given as follows: [13].

$$
\begin{gathered}
P_{f a}=P\left(Y>\gamma \mid \mathrm{H}_{0}\right)=Q\left(\frac{\gamma-\sigma_{n}^{2}}{\sigma_{n}^{2} / \sqrt{N}}\right) \\
P_{d}=P\left(Y>\gamma \mid \mathrm{H}_{1}\right)=Q\left(\frac{\gamma-\sigma_{n}^{2}(1-S N R)}{\sigma_{n}^{2} / \sqrt{N} \cdot \sqrt{2 \cdot S N R+1}}\right)
\end{gathered}
$$

where $Q(x)$ is defined by the complementary distribution function of the standard Gaussian and is given as

$$
Q(x)=\frac{1}{2 \pi} \int_{x}^{\infty} e^{-u^{2} / 2} d u
$$

$S N R$ is a signal to noise ratio, which can be defined in terms of the signal and noise variance $\left(\sigma_{s}^{2}\right.$ and $\left.\sigma_{n}^{2}\right)$ as

$$
S N R=\frac{\sigma_{S}^{2}}{\sigma_{n}^{2}} .
$$

Therefore for a constant false alarm rate $\left(P_{f a}\right)$ the threshold is given by [13]

$$
\gamma=\delta_{n}^{2}+\frac{Q^{-1}\left(P_{f a}\right) \cdot \delta_{n}^{2}}{\sqrt{N}} .
$$




\section{CoOperative SEnsing Fusion Rules}

To improve signal detection, the cooperative spectrum sensing can be used [14], [15]. In the prospective scenario, there are $M$ SUs that sense one PU. Each of the SUs makes its own decision regarding the presence or absence of the $\mathrm{PU}$, and forwards the binary decision ( 1 or 0$)$ to a fusion center (FC) for further processing. SUs are located at a negligible distance from each other, compared to the distance from them to PU. Thus from a long-term perspective the primary signal received by all the SUs has the same local mean signal power. For simplicity, we have first assumed that the noise, fading statistics and average SNR are the same for each SU, and the channels between SUs and FC are ideal (i.e. there is no loss of information). A final decision on the presence of PU is made by $k$ out of $M$ SUs and can be described by a binomial distribution based on the Bernoulli trials where each trial represents the decision process of each SU. The generalized formula for the probability of detection at the fusion center is given by [14], [15]:

$$
\begin{aligned}
P_{f a} & =\sum_{\mathrm{l}=\mathrm{k}}^{\mathrm{M}}\left(\begin{array}{l}
M \\
l
\end{array}\right) P_{f a, i} l\left(1-P_{f a, i}\right)^{M-l} \\
P_{d} & =\sum_{l=\mathrm{k}}^{\mathrm{M}}\left(\begin{array}{l}
M \\
l
\end{array}\right) P_{d, i} l\left(1-P_{d, i}\right)^{M-l}
\end{aligned}
$$

where $P_{d, i}$ and $P_{f a, i}$ are the probabilities of detection and false alarm, respectively, for each SU. In this paper three rules of the hard combination scheme are discussed.

\section{A. Logical OR-Rule}

In this rule, a decision that the PU is present is made if any of the SUs detects the PU. The cooperative probability of detection (false alarm) using $O R$ fusion rule can be evaluated by setting $k=1$ in eq. $(14,15)$ :

$$
\begin{gathered}
P_{f a}=1-\prod_{l=1}^{M}\left(1-P_{f a, i}\right) \\
P_{d}=1-\prod_{l=1}^{M}\left(1-P_{d, i}\right) .
\end{gathered}
$$

\section{B. Logical AND-Rule}

In this rule, a decision that the PU is present is made if all SUs have detected the PU. The cooperative probability of detection (false alarm) using $A N D$ fusion rule can be evaluated by setting $k=M$ in eq. $(14,15)$ :

$$
\begin{aligned}
P_{f a} & =\prod_{l=1}^{M} P_{f a, i} \\
P_{d} & =\prod_{l=1}^{M} P_{d, i} .
\end{aligned}
$$

\section{Logical MAJORITY-Rule}

In this rule, a decision that the PU is present is made if half or more of the SUs detect the PU. The cooperative probability of detection (false alarm) using MAJORITY fusion rule can be evaluated by setting $k=M / 2$ in eq. (14, 15):

$$
P_{f a}=\sum_{\mathrm{l}=\mathrm{M} / 2}^{\mathrm{M}}\left(\begin{array}{l}
M \\
l
\end{array}\right) P_{f a, i} l\left(1-P_{f a, i}\right)^{M-l}
$$

$$
P_{d}=\sum_{1=\mathrm{M} / 2}^{\mathrm{M}}\left(\begin{array}{l}
M \\
l
\end{array}\right) P_{d, i}{ }^{l}\left(1-P_{d, i}\right)^{M-l}
$$

\section{TESTBED DESCRIPTION}

We use a similar measurement setup as the one used in our previous paper [16]. Fig. 2 depicts this setup.

It consists of one personal computer $(P C)$ and two USRP 2 devices. One of the USRP2 is carrying out $S U_{1}$ as well as $P U$ roles and is connected to the PC through a Gigabit Ethernet port.

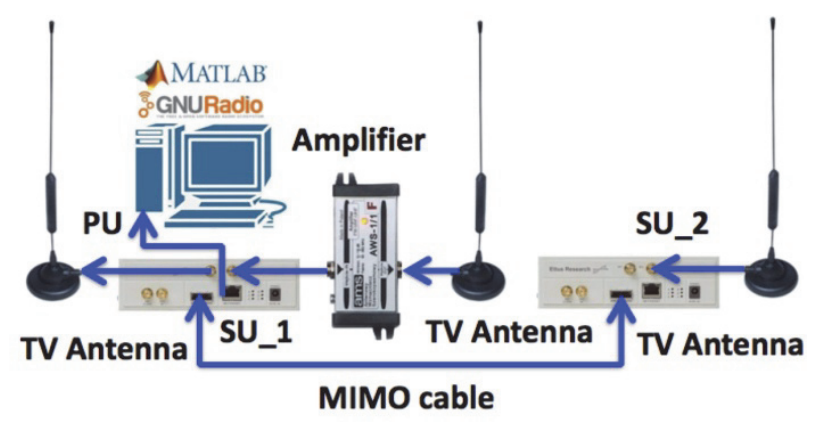

Fig. 2. Experimental setup working in TV whitespaces.

The second USRP2 (which is carrying out a $S U_{2}$ role) is connected to the first USRP through a MIMO cable to synchronize the system. Note that the precise synchronization between the SUs must be realized in order to realize the cooperative scheme. Optional GPSdisciplined oscillators provide the capability to synchronize devices to the GPS standard over a large geographic area [17].

In the setup, the first USRP implementing the $S U_{l}$ and $P U$ employs the $W B X$ daughter board (front-end), while the $S B X$ front-end is used in $S U_{2}$ device. One $S U$ had an antenna behind the window and it was connected with an amplifier to enhance the received signal, the other had it on the laboratory table and the distance between them was about 4 meters. With such a scenario, two received-signal replicas were created.

Both USRPs work in the TV whitespace. The primary user signal, which was transmitted by the first USRP, is an 8PSK signal with a bandwidth of $200 \mathrm{kHz}$ (bandwidth corresponding to wireless microphones bandwidth, although the modulations are different) centered at 611 $\mathrm{MHz}$ carrier frequency, which corresponds to one of the TV channels (in our experiment the $38^{\text {th }}$ channel). A priori we know that in the measurement location this channel is vacant. Each $S U$ scans two channels, the first one is a free channel at $610 \mathrm{MHz}$ frequency (this channel is vacant throughout the experiment), the second one is a channel where PU transmits. Note that although the channel is vacant, it is possible to suppose that the character of received signal is not exactly white Gaussian as it can contain interferences from neighboring bands.

The $P C$ is running Fedora 16, the signal processing application is done using an open-source GNU Radio version 3.7.2.1. Each of $S U s$ sensed the channel of interest. By applying the GNU Radio the sensing results are recorded into the data files for subsequent processing. After that based on the recorded data files, the values of $P_{d}$ 
and $P_{f a}$ are calculated for each $S U$ in Matlab in order to estimate corresponding ROC curves. The equations to estimate these quantities are as follows:

$$
\begin{aligned}
P_{d} & =\frac{\text { Number of segments that have } Y>\gamma \mid \mathrm{H}_{1}}{\text { Number of observed segments }} \\
P_{f a} & =\frac{\text { Number of segments that have } Y<\gamma \mid \mathrm{H}_{1}}{\text { Number of observed segments }}
\end{aligned}
$$

In accordance with fusion rules for cooperative sensing, a final decision about a vacant channel is made. The most important results are presented below.

\section{EXPERIMENTAL RESULTS}

To evaluate the performance of a particular method of (cooperative) spectrum sensing techniques, the key characteristics as $P_{d}$ and $P_{f a}$ are used [3]. The ROC curve graph visually shows the dependence between these two parameters. All calculations for constructing diagrams are performed in Matlab.

First, we analyzed the dependence of $P_{d}$ and $P_{f a}$ on the SNR values. Fig. 3 demonstrates a higher detection reliability for increased SNR values.

Fig. 4 is related to the practical implementation of K-S testing device and describes ROC curves for different numbers of points used for empirical and theoretical CDF approximation $\left(N_{C D F}\right)$.

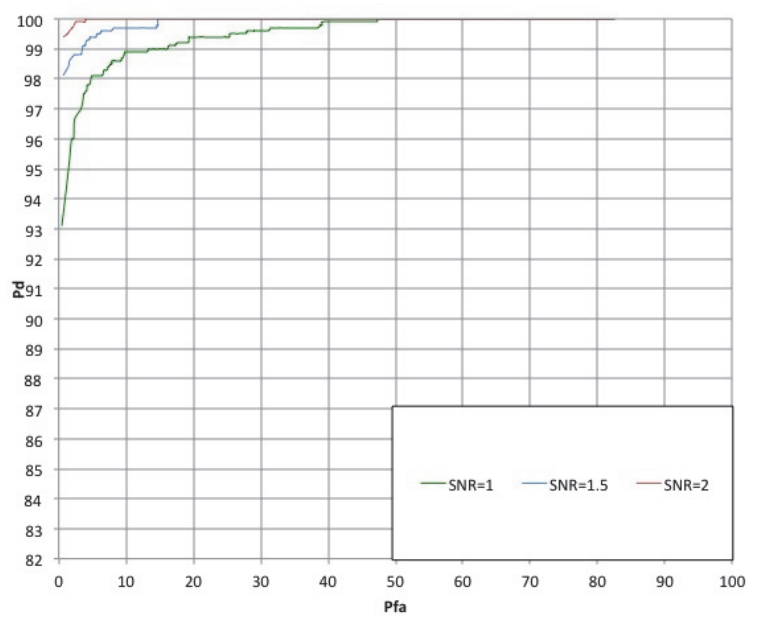

Fig. 3. ROC curves for 8PSK signal for different SNR, 1000 segments with 100 data samples.

It can be observed that also the value of $N_{C D F}=7$ provides sufficient reliability only slightly degraded than in the case of a higher number of points for approximation. Note that in this case the number of points in each signal segment and SNR value is fixed.

To illustrate the meaning of $N_{C D F}$ and its influence on the empirical CDF, we present Fig. 5. It can be seen that with increasing $N_{C D F}$ the empirical CDF becomes smoother and closer to the theoretical one (in the case of valid hypothesis $\mathrm{H}_{0}$ ).

Fig. 6 shows simulation results for different channels it compares the theoretical AWGN case with the real measured channel in TV band. As expected and evident from the figure, the pure AWGN channel results in a higher detection probability $P_{d}$ than the real fading channel.

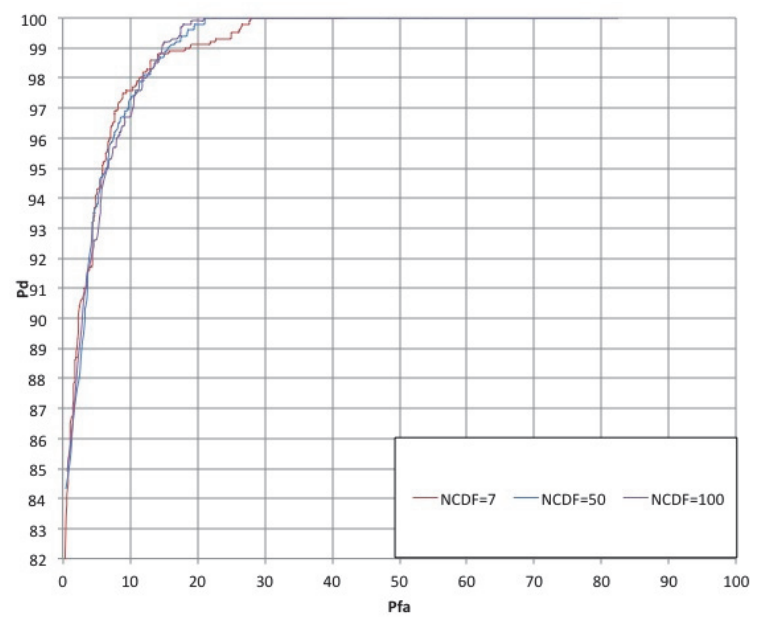

Fig. 4. ROC curves for 8PSK signal for different $N_{C D F}$, $\mathrm{SNR}=0.5 \mathrm{~dB}, 1000$ segments with 100 data samples.

Note here that ROC curve evaluation has been done for one hundred samples per segment only. This relatively small value of the number of samples per segment (in comparison with e.g. 1000-5000 samples in [2]) results in a lower SNR performance.
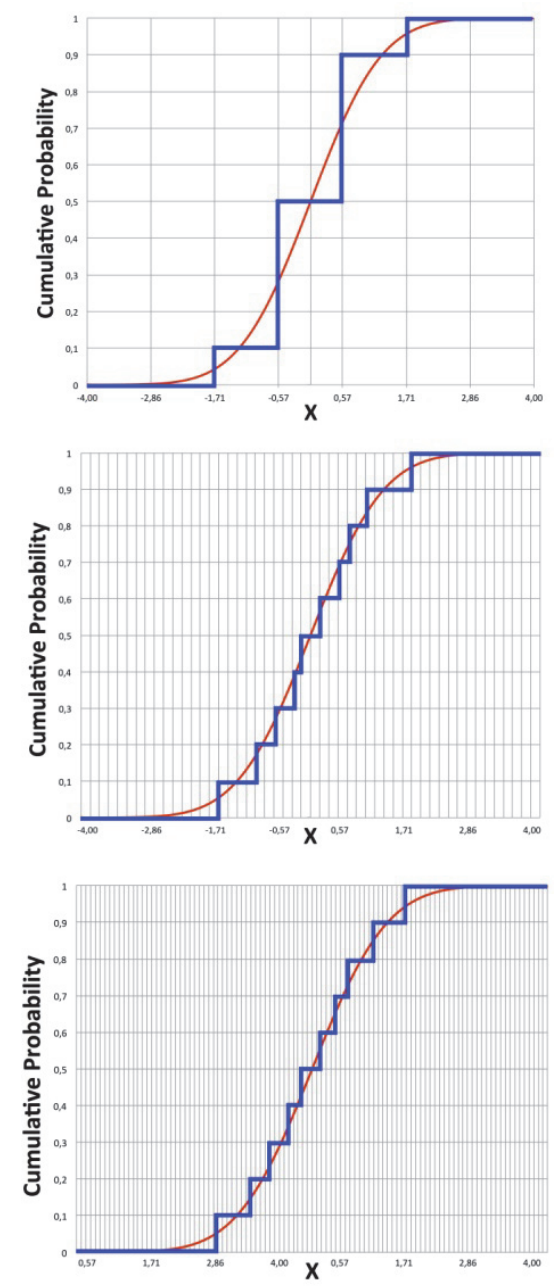

Fig. 5. Empirical (blue line) and theoretical (red line) CDFs for different $N_{C D F}$ (top $N_{C D F}=7$, in the middle $N_{C D F}=50$, bottom $N_{C D F}=100$ ).

Finally, we compared the single spectrum sensing by each SU with their cooperative version in accordance with 
three most-used fusion rules. Fig. 7 and Fig. 8 illustrate these comparisons for K-S test and ED, respectively.

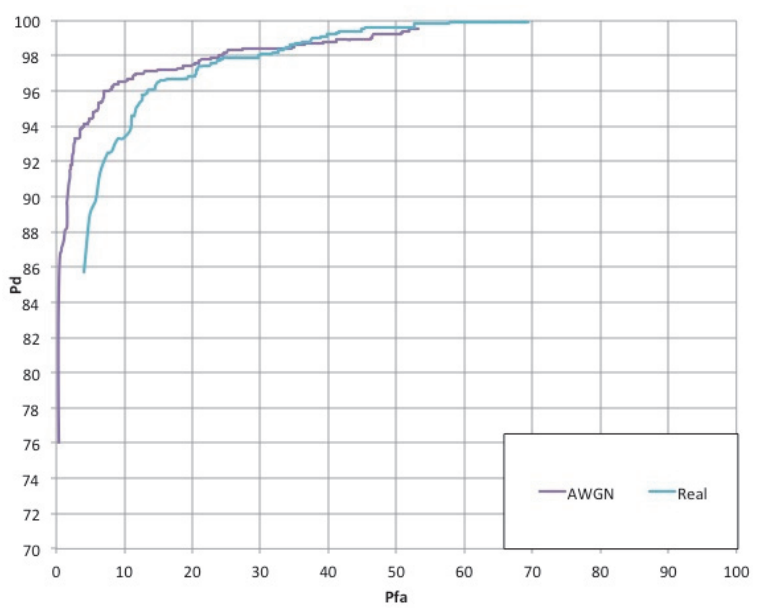

Fig. 6. ROC curves for 8PSK signal for different channel, $\mathrm{SNR}=9 \mathrm{~dB}, 1000$ segments with 100 data samples.

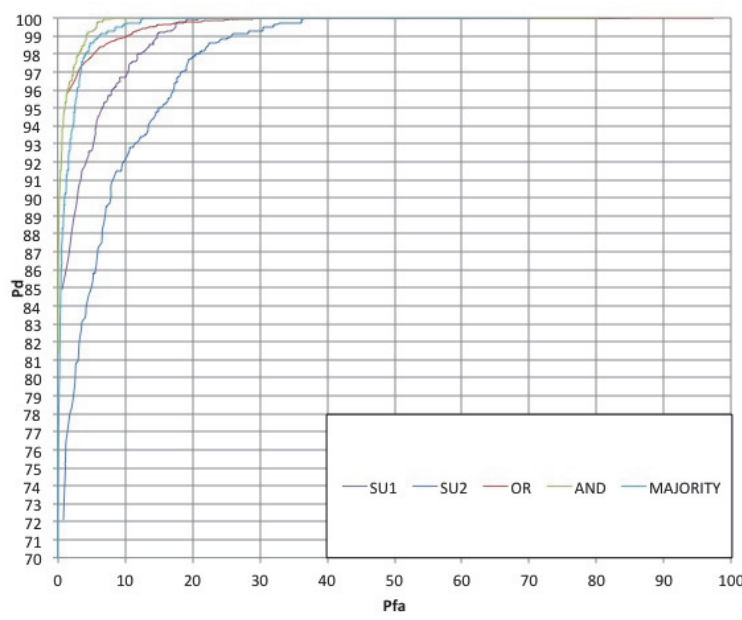

Fig. 7. ROC curves for KS test for 8PSK signal for three fusion rules, $\mathrm{SNR}=0.5 \mathrm{~dB}, 1000$ segments with 100 data samples.

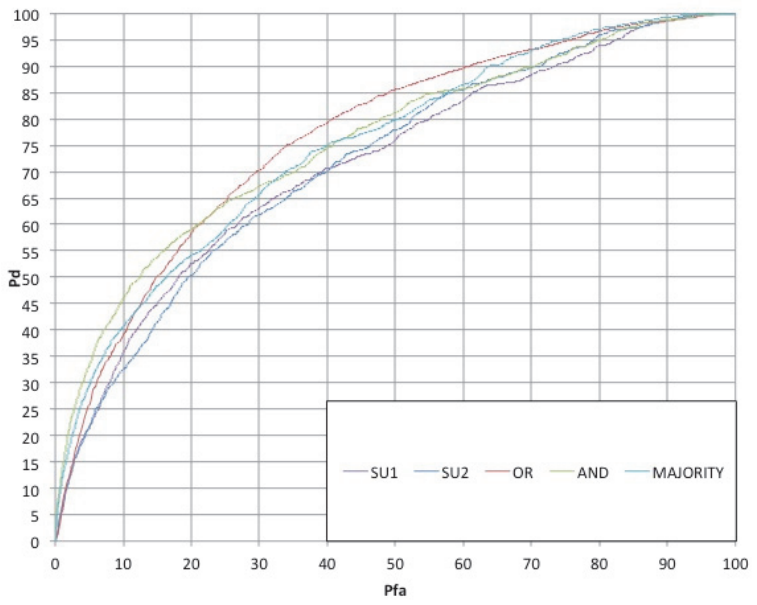

Fig. 8. ROC curves for ED for 8PSK signal for three fusion rules, $\mathrm{SNR}=0.5 \mathrm{~dB}, 1000$ segments with 100 data samples.

As can be seen from Figs. 7 and 8, all cooperative fusion rules gave a better detection performance than the individual sensing. The $A N D$ rule has a better detection performance than the $O R$ and MAJORITY rules at low $P_{f a}$.
This is due to the fact that for $O R$ rule the FC decides whether a PU is present when at least one SU detects it. While in the $A N D$ rule all SUs must detect a PU signal. Also worth noting is the fact that K-S test has a better detection performance than ED as for individual sensing as well as for all cooperative fusion rules.

As it is known [18], for the IEEE 802.22 standard $P_{d}$ should be $90 \%$ or higher at $P_{f a}=0.1$. In our experiment, all fusion rules and each $\mathrm{SU}$ for $\mathrm{K}-\mathrm{S}$ test meet this requirement, whereas $\mathrm{ED}$ cannot reach it for the same SNR values.

\section{CONCLUSION}

In this paper we present a performance evaluation of Kolmogorov-Smirnov test-based and energy detectionbased spectrum sensing methods in close-to-real channel conditions. We have compared the influence of real channel noise and interferences on the sensing performance expressed by the Receiver Operational Characteristics and the effect of cumulative function approximations by a finite number of samples. Finally, the hard (binary) fusion rules for cooperative sensing were used to demonstrate their improvement on the decision process. Using an example of the sensed 8PSK signal with different SNR values, it is first checked that the performance of individual sensing improves with an increased SNR. It was also demonstrated that the probability of detection for real channel deteriorates in comparison with AWGN usually assumed in theoretical analysis. On the contrary, the effect of finite approximation of distribution functions seems to be marginal.

In our future work, we will use the investigated sensors for TV and wireless microphones signals cooperative sensing in a city area on several vacant TV channels.

\section{REFERENCES}

[1] IEEE 802.22 Working Group on Wireless Regional Area Networks. Enabling Rural Broadband Wireless Access Using Cognitive Radio Technology in TV Whitespaces. Recipient of the IEEE SA Emerging Technology Award. [Online] Available: http://grouper.ieee.org/groups/802/22/ (May 31, 2014).

[2] R. Marsalek, K. Povalac, "Kolmogorov - Smirnov test for spectrum sensing: from the statistical test to energy detection," IEEE Workshop on Signal Processing Systems, pp. 97-102, 2012.

[3] D. Teguig, B. Scheers and V. Le Nir, "Data fusion schemes for cooperative spectrum sensing in cognitive radio networks," Military Communications and Information Systems Conference (MCC), pp. $1-7,2012$.

[4] Md. Shamim Hossain, et. al., "Hard Combination Data Fusion for Cooperative Spectrum Sensing in Cognitive Radio," in International Journal of of Elektrical and Computer Engineering (IJECE), vol. 2, No.6, December 2012, pp. 818-818.

[5] H. Du, et. al., "Hybrid Cooperative Spectrum Sensing Scheme Using Double-Fusion in Cognitive Radio Networks," in Journal of Computational Information Systems, vol. 7, 2011, pp. 1500-1507.

[6] S. Chaudhari, et al., "Measurement Campaign for Collaborative Sensing using Cyclostationary Based Mobile Sensors," IEEE International Symposium on Dynamic Spectrum Access Networks (DYSPAN), pp. 283-290, 2014.

[7] S. Kyperountas, et al., "A Comparison of Fusion Rules for Cooperative Spectrum Sensing in Fading Channels," Virginia Tech Symposium on Wireless Personal Communications, pp. 1-6, 2010.

[8] A. Gameiro, et. al., "Selective reporting - a half signalling load algorithm for distributed sensing," in EURASIP Journal on Wireless Communications and Networking, vol. 6, 2013, pp. 1-14. 
[9] K. Povalac, R. Marsalek, "Application of the spectrum sensing based on the Kolmogorov - Smirnov test to the OFDM resource allocation," in Recent Researches in Circuits, Systems, Communications \& Computers - Proc. of 2nd European Conference of Communications (ECCOM'11), 2011, pp. 89-93.

[10] H. Wang, "A Spectrum Sensing Algorithm Based on Kolmogorovsmirnov Two-sample Test," in Journal of Computational Information Systems, vol. 8, 2012, pp. 2601-2607.

[11] W. Conover, Practical Nonparametric Statistics, 3rd Edition, John Wiley and Sons, 1999, 592 p. ISBN: 978-0- 471-16068-7.

[12] S. M. Kay, Intuitive Probability and Random Processes Using MATLAB, New York, USA: Springer, 2006, ISBN 978-0-38724157-9.

[13] P.R. Nair, A.P. Vinod and A.K. Krishna, "An adaptive threshold based energy detector for spectrum sensing in cognitive radios at low SNR," IEEE International Conference on Communication Systems (ICCS), pp. 574 - 578, 2010.

[14] Yang, X. and Fei, H., Cognitive Radio Networks, 2nd Ed. Taylor \& Francis Group, LLC, 2009.
[15] D. Ruilong, et al., "Energy-Efficient Cooperative Spectrum Sensing by Optimal Scheduling in Sensor-Aided Cognitive Radio Networks," IEEE Transactions on Vehicular Technology, vol. 61, pp. $716-725,2012$.

[16] D. Lekomtcev, R. Marsalek, "USRP setup for energy detectionbased Cooperative Spectrum Sensing for Cognitive Radio Networks," IEEE Konference Zvi̊le 2014, pp. 41-44, 2014.

[17] Application Note Synchronization and MIMO Capability with USRP Devices Ettus Research. [Online] Available: http://www.ettus.com/content/files/kb/mimo_and_sync_with_usrp.p df (May 31, 2014)

[18] "IEEE Std 802.22 $2^{\mathrm{TM}}$-2011 IEEE Standard for Information Technology - Telecommunications and information exchange between systems Wireless Regional Area Networks (WRAN) Specific requirements - Part 22: Cognitive Wireless RAN Medium Access Control (MAC) and Physical Layer (PHY) Specifications: Policies and Procedures for Operation in the TV Bands," 2011. 